\title{
In-process automatic wavelength calibration for CCD-spectrometers
}

\author{
J. Mirapeix, A. Cobo, A.M. Cubillas, O.M. Conde, J.M. Lopez-Higuera \\ Photonics Engineering Group, Univ. de Cantabria, Avda. Los Castros s/n, 39005 Santander, Spain
}

\begin{abstract}
In CCD-spectrometers, the relation between the CCD-pixel number and the associated wavelength is established by means of a calibration polynomial, whose coefficients are typically obtained using a calibration lamp with known emission line wavelengths and a regression procedure. A recalculation of this polynomial has to be performed periodically, as the pixel number versus wavelength relation can change with ambient temperature variations or modifications in the optics attached to the spectrometer connector. Given that this calibration procedure has to be performed off-line, it implies a disturbance for industrial scenarios, where the monitoring setup must be altered.

In this paper an automatic wavelength calibration procedure for CCD-spectrometers is proposed. It is based on a processing scheme designed for the in-process estimation of the plasma electronic temperature, where several plasma emission lines are identified for each spectral capture. This identification stage involves the determination, by means of a sub-pixel algorithm, of the central wavelength of those lines, thus allowing an on-line wavelength calibration for each single acquired spectrum. The proposed technique will be demonstrated by means of several experimental arc-welding tests.
\end{abstract}

Keywords: arc-welding, on-line monitoring, plasma optical spectroscopy, CCD-spectrometer, wavelength calibration

\section{INTRODUCTION}

CCD spectrometers are used in a wide range of different spectroscopic techniques, some of them devoted to the inspection of industrial processes, like food, tobacco or weld quality monitoring, among others. Both compact dimensions and low cost are important features of these devices. However, when the requirements in terms of optical resolution are significant, periodic calibrations have to be performed to avoid ambiguous or unexpected results.

A good example can be found in the monitoring of welding processes, where on-line inspection is carried out in an attempt to identify the appearance of weld defects. Plasma optical spectroscopy has proved to be a suitable approach, as there exists a correlation between some spectroscopic parameters and the quality of the seams [1,2]. The correct identification of the atomic emission lines participating in the plasma is a key stage in this process, which will obviously depend on a correct spectrometer calibration. It is common practice to perform an initial wavelength calibration by the manufacturer of the spectrometer, where the pixel number of the CCD is associated with the corresponding wavelength or spectral band. This task can be fulfilled by means of a calibration lamp, which is a light source providing emission lines of known central wavelengths. In this regard, by performing a regression procedure, a calibration polynomial is obtained. However, this polynomial needs to be recalculated as the pixel number versus wavelength relation exhibits dependencies on the ambient temperature or the input optics attached to the spectrometer.

If a calibration lamp or any other light source is needed for the calibration process, it implies that this process will most likely have to be carried out off-line. When an industrial monitoring system is considered, this calibration procedure could be an issue in terms of the process productivity, as it will force to stop at least the monitoring system itself. All in all, it seems clear that it would be desirable to perform this calibration procedure while the process is being analyzed by the system, and without including any external device, thus saving the costs involved.

In previous works, we analyzed how to improve the stability of the plasma electronic temperature $T_{e}$ profiles by involving in the processing scheme sub-pixel algorithms [3]. This approach allows a more accurate estimation of the central wavelengths of the chosen plasma emission lines, which is the key parameter to perform an unambiguous identification of them. To identify a specific plasma emission line means to associate to it its corresponding chemical species and ionization stage. This process is typically carried out designing a local database with the spectroscopic information of the species participating in the plasma, which can be obtained from the NIST atomic spectra database [4]. Figure 1 shows the processing blocks considered for the estimation of $T_{e}$ in [3] (and the additional block devoted to

Optical Sensors 2008, edited by Francis Berghmans, Anna Grazia Mignani, Antonello Cutolo, Patrick P. Meyrueis, Thomas P. Pearsall, Proc. of SPIE Vol. 7003, 70031T, (2008) · 0277-786X/08/\$18 · doi: 10.1117/12.781040 
spectral calibration), and Figure 2 depicts a schematic representation of the identification process. Given that in this process the central wavelengths of the chosen emission lines are known, this information can be used to carry out an automatic, and therefore on-line, spectral calibration of a CCD-spectrometer.

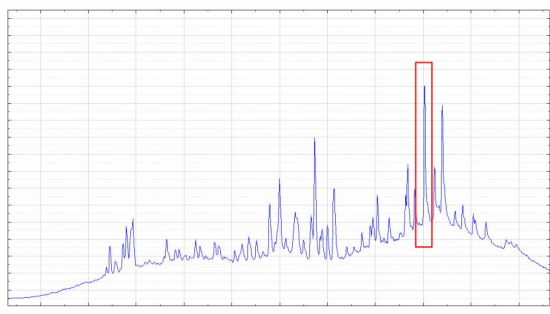

Welding plasma spectrum

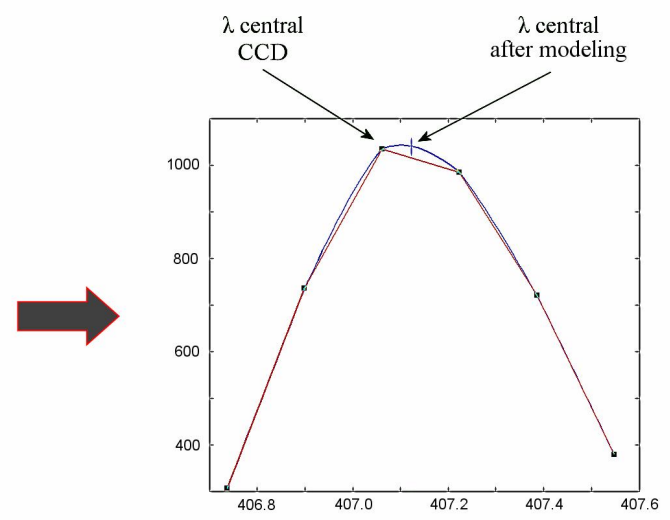

Peak modeling

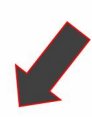

\begin{tabular}{|c|c|c|c|c|c|c|}
\hline spec. & $\begin{array}{l}\text { wavelength } \\
\text { vacuum } \\
(\mathrm{nm})\end{array}$ & $\begin{array}{l}\text { Rel. } \\
\text { Int: }\end{array}$ & $(10 \wedge 85-1)$ & $A C C$ & (ev) & $\left.\begin{array}{c}\mathrm{Ek} \\
(\mathrm{eV})\end{array}\right)$ \\
\hline $\begin{array}{lll}0 & \text { III } \\
0 & \text { III } \\
0 & \text { II } \\
0 & \text { III } \\
0 & \text { III }\end{array}$ & $\begin{array}{l}150.1633 \\
150.2886 \\
150.3068 \\
150.4076 \\
150.4123\end{array}$ & $\begin{array}{l}15 \\
10 \\
12\end{array}$ & $\begin{array}{l}1.69 \mathrm{e}-01 \\
4.99 \mathrm{e}-01 \\
5.53 \mathrm{e}-02 \\
2.81 \mathrm{e}-01 \\
5.56 \mathrm{e}-01\end{array}$ & $\begin{array}{l}c- \\
c+ \\
c+ \\
c- \\
c+\end{array}$ & $\begin{array}{l}45.26003 \\
20.57995 \\
20.58095 \\
45.99944 \\
20.58095\end{array}$ & $\begin{array}{l}54.18266 \\
=\quad 28.82969 \\
=\quad 58.82969 \\
=\quad 28.18266 \\
\end{array}$ \\
\hline \begin{tabular}{|l|l|}
0 IV \\
0 IV \\
0 IV \\
0 VII \\
0 IV
\end{tabular} & $\begin{array}{l}151.396 \\
151.8799 \\
151.902 \\
152.161 \\
152.281\end{array}$ & & $\begin{array}{l}1.17 e+00 \\
9.67 e-01 \\
1.93 e-01 \\
2.150 e+0 \\
1.97 e+00\end{array}$ & $\begin{array}{l}\text { E } \\
\text { B } \\
\text { A } \\
\text { AA } \\
B\end{array}$ & $\begin{array}{r}64.3117 \\
64.3105 \\
64.3117 \\
712.689 \\
68.1655\end{array}$ & $\begin{array}{l}-72.5012 \\
=72.4739 \\
=72.4739 \\
-720.838\end{array}$ \\
\hline $\begin{array}{cl}0 & \text { IV } \\
0 & \text { IV } \\
\text { O IV } & \text { XIV } \\
0 & \text { VII } \\
0 & \text { III } \\
0\end{array}$ & $\begin{array}{l}152.427 \\
1552.495 \\
152.800 \\
153.304 \\
153.479\end{array}$ & & $\begin{array}{l}3.94 \mathrm{e}-01 \\
2.36+00 \\
1.9 \mathrm{e}-07 \\
1.099 \mathrm{e}+1 \\
\text { 2. } 81 \mathrm{e}-02\end{array}$ & $\begin{array}{l}\text { B } \\
\text { B } \\
\text { E } \\
\text { AA } \\
\text { D }\end{array}$ & $\begin{array}{c}68.1734 \\
68.1734 \\
37.1720 \\
712.717 \\
43.39752\end{array}$ & $\begin{array}{l}=76.307 \\
=76.3037 \\
=45.2162 \\
=720.804 \\
51.475\end{array}$ \\
\hline
\end{tabular}

\section{Search based in a local database}

Fig. 1. Schematic diagram of the required steps to perform a plasma emission line identification.

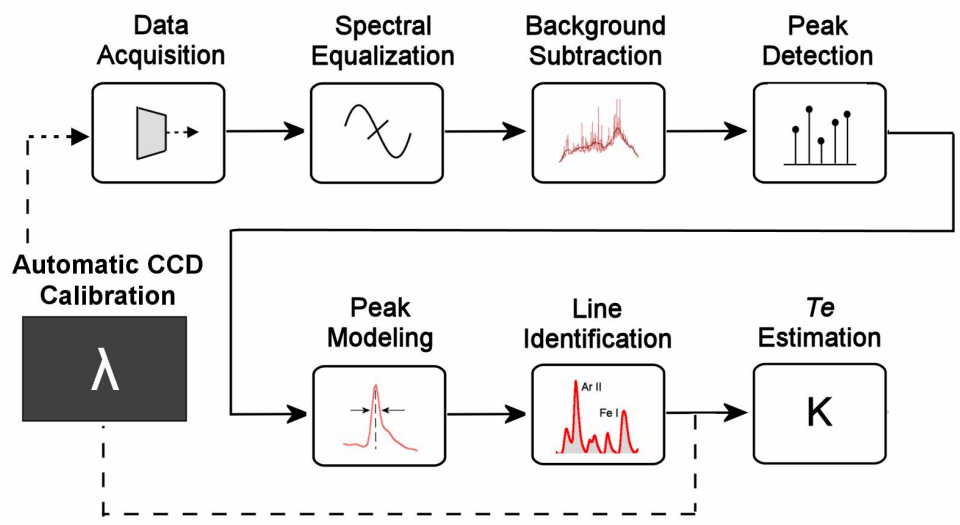

Fig. 2. Proposed processing scheme for on-line weld quality monitoring based on the estimation of the plasma electronic temperature and additional automatic ccd-spectrometer spectral calibration. 


\section{SPECTRAL CALIBRATION OF CCD SPECTROMETERS}

The typical approach considered to relate the pixel number of a CCD spectrometer to its corresponding wavelength is based on the use of a polynomial regression. In this regard, a calibration polynomial will be obtained such as

$$
\lambda(p)=C_{0}+C_{1} p+C_{2} p^{2}+C_{3} p^{3},
$$

where $\lambda$ is the wavelength, $p$ the pixel number and $C_{0}$ to $C_{3}$ the polynomial coefficients to determine. It is worth mentioning that the polynomial order could be adjusted in terms of the specific requirements, but typically second and third orders are chosen. The number of known emission lines used in the calibration process will have to exceed the polynomial order, and obviously the process will be improved by considering more lines, although this will also imply a cost in terms of computational performance (on-line) or calculations (off-line). The unknown coefficients of equation (1) can be calculated by trying to minimize the following expression:

$$
S=\sum_{i=0}^{m}\left[\lambda\left(p_{i}\right)-y_{i}\right]^{2},
$$

where $y_{i}$ are the known wavelengths of the emission lines chosen for calibration. This method is called linear least squares regression, and considering the partial derivatives of $S$ with respect to the coefficients and equalling to zero, we come to the following expression:

$$
\left(\begin{array}{cccc}
n & \sum_{i=1}^{n} p_{i} & \sum_{i=1}^{n} p_{i}^{2} & \sum_{i=1}^{n} p_{i}^{3} \\
\sum_{i=1}^{n} p_{i} & \sum_{i=1}^{n} p_{i}^{2} & \sum_{i=1}^{n} p_{i}{ }^{3} & \sum_{i=1}^{n} p_{i}{ }^{4} \\
\sum_{i=1}^{n} p_{i}^{2} & \sum_{i=1}^{n} p_{i}^{3} & \sum_{i=1}^{n} p_{i}^{4} & \sum_{i=1}^{n} p_{i}{ }^{5} \\
\sum_{i=1}^{n} p_{i}{ }^{3} & \sum_{i=1}^{n} p_{i}{ }^{4} & \sum_{i=1}^{n} p_{i}{ }^{5} & \sum_{i=1}^{n} p_{i}{ }^{6}
\end{array}\right) \cdot\left(\begin{array}{l}
C_{0} \\
C_{1} \\
C_{2} \\
C_{3}
\end{array}\right)=\left(\begin{array}{c}
\sum_{i=1}^{n} y_{i} \\
\sum_{i=1}^{n} y_{i} p_{i} \\
\sum_{i=1}^{n} y_{i} p_{i}{ }^{2} \\
\sum_{i=1}^{n} y_{i} p_{i}{ }^{3}
\end{array}\right) .
$$

The Gauss-Jordan method can be applied to find the desired polynomial coefficients [5]. Figure 3 shows an example of the spectrum provided by a commercial calibration lamp (Ocean Optics CAL2000). The central wavelength of the emission lines in the figure are known and provided by the manufacturer.

As previously commented in Section 1, during the processing stages designed for an on-line weld quality monitoring system some plasma emission lines are identified, typically those exceeding a specific intensity threshold. After the identification stage, the central wavelength of those lines is also known, thus allowing their use as an "internal" calibration lamp. Figure 4 shows an example of a plasma spectrum captured during an experimental weld test. It can be observed that the plasma radiation is typically formed by a background (continuum) signal and the contribution of several emission lines. Although only four iron neutral atom emission lines are highlighted in figure 4, it should be mentioned that almost all the lines observed in the plasma radiation could be identified, but in this case the signal-tonoise threshold in the line selection process was increased. The known lines at the output of the identification process can be used for calibration purposes. 


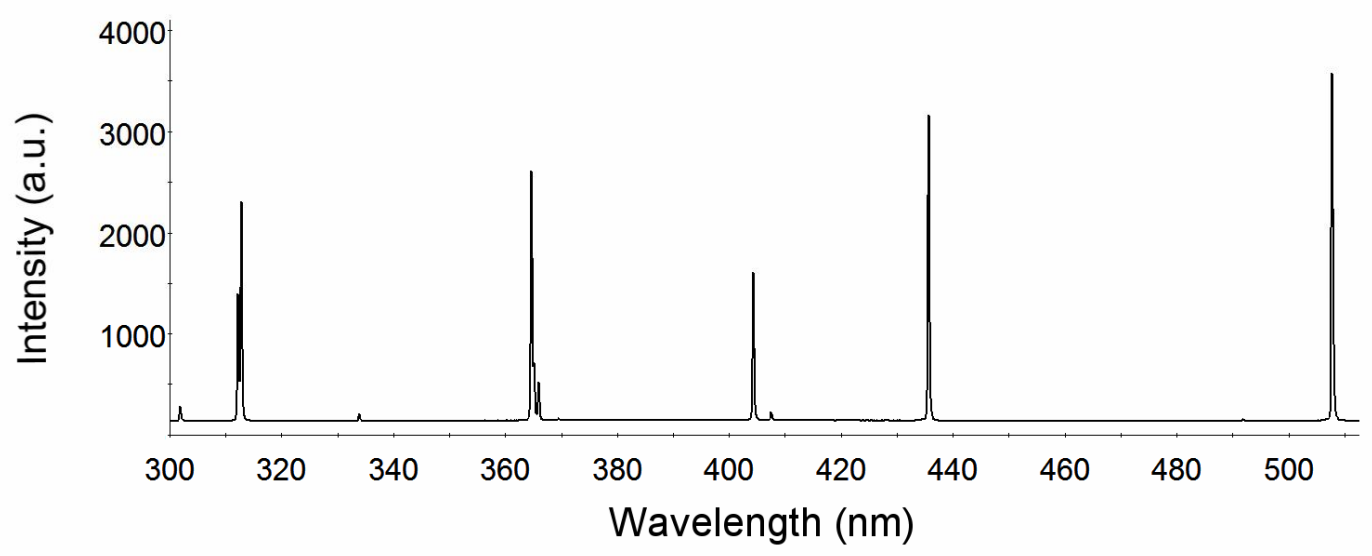

Fig. 3. Example of spectrum provided by a commercial calibration lamp.

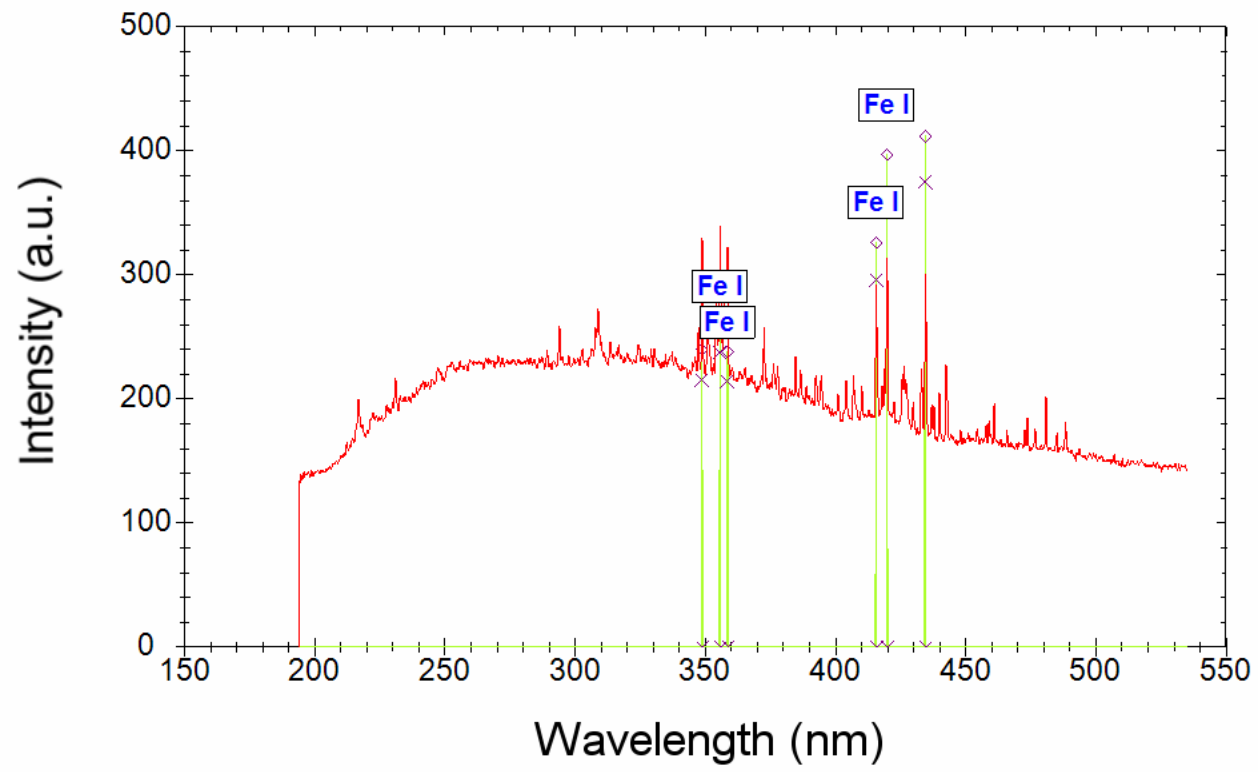

Fig. 4. Example of a plasma spectrum captured during an experimental weld test with line identification.

\section{EXPERIMENTAL ISSUES}

Several experimental welding tests were performed in the laboratory to check the feasibility of the proposed technique. The setup was formed by a TIG welding system (Kemppi Mastertig 2200 power source) and a high-precision positioning system (Newport MM4005 and MTM100PP1) controlled via PC. The AISI-304 stainless steel plates were attached to the positioning system, as the welding torch was kept fixed. Argon was used as the protection gas needed in this variety of arc-welding process, with a flow rate of $12 \mathrm{~L} / \mathrm{min}$. An Ocean Optics USB2000 CCD-spectrometer was chosen, with 2048 pixels and a spectral range from 195 to $535 \mathrm{~nm}$.

An initial study was conducted on the validity of the polynomial provided by the manufacturer. A Gaussian fit was performed to any reference line considered in the calculations by means of the commercial software PeakFit [6]. The 
resulting polynomial compared to the original one is presented in Table 1, including the mean error. The lines provided by the CAL2000 and used in the process are listed in Table 2, and Table 3 shows the particular error in both cases for all the CAL2000 lines considered (figure 5 depicts the mean error associated to each polynomial in terms of the calibration emission lines). It should be mentioned that the measured error is smaller for all the lines with the exception of the $\mathrm{Hg}$ line located at $365.015 \mathrm{~nm}$.

Table 1. Comparison of manufacturer and Gaussian-fitted polynomials.

Polynomial coefficients and mean error

\begin{tabular}{lcccc}
\multicolumn{5}{l}{ Manufacturer coefficients } \\
\hline $\mathrm{C}_{0}$ & $\mathrm{C}_{1}$ & $\mathrm{C}_{2}$ & $\mathrm{C}_{3}$ & Mean error $\left(\mathrm{nm}^{2}\right)$ \\
\hline 193.953841 & 0.189284 & $-1.110 \mathrm{E}-05$ & 0 & 0.049758 \\
\\
\multicolumn{7}{l}{ Coefficients after Gaussian fitting } & & & \\
\hline $\mathrm{C}_{0}$ & $\mathrm{C}_{1}$ & $\mathrm{C}_{2}$ & $\mathrm{C}_{3}$ & Mean error $\left(\mathrm{nm}^{2}\right)$ \\
\hline 194.947413 & 0.186984 & $-9.0745 \mathrm{E}-06$ & $-6.630 \mathrm{E}-10$ & 0.002790 \\
\hline
\end{tabular}

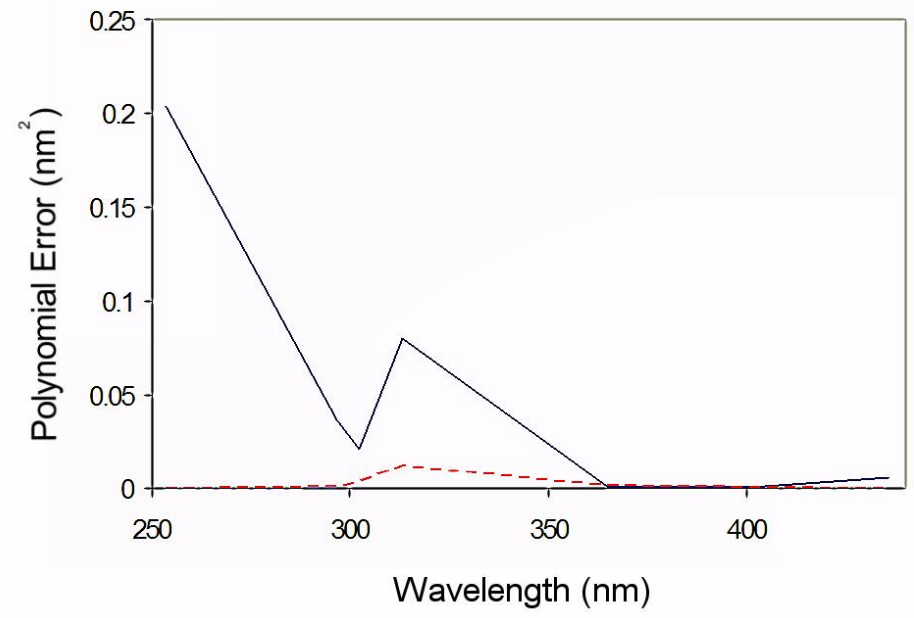

Fig. 5. Manufacturer and Gaussian-fitted polynomial errors.

Table 2. Hg lines provided by the CAL2000.

\begin{tabular}{c}
\hline Peak Wavelength $(\mathrm{nm})$ \\
\hline 253.652 \\
296.728 \\
302.15 \\
313.155 \\
365.015 \\
404.656 \\
435.833 \\
\hline
\end{tabular}

Different studies were performed to evaluate the performance of the designed procedure to fulfill an on-line automatic spectral calibration of the chosen CCD-spectrometer. Basically two parameters were modified during the tests: the number of plasma emission lines involved in the required regression and the number of spectral captures where a new polynomial was obtained. In terms of on-line inspection, the best moment to carry out the automatic calibration is the beginning of the process. However, the inherent instability of arc-welding process at this moment makes it difficult to 
find suitable emission lines to be used, as this instability is transferred to the plasma radiation. This can be observed in the results presented in Table 3. In the case of Weld test $n^{\circ} 1$, the worst result is obtained when 20 spectral lines and spectral samples 0 to 10 are considered. It is worth noting that the mean error has not been calculated involving only the spectral samples where the polynomial is recalculated, but all the samples associated with a seam are considered. In this regard, once the automatic calibration is over, i.e. the upper limit of the sample window provided has been exceeded, the optimum polynomial in terms of the resulting error is used during the rest of the process. It can be observed that the minimum error for Weld test $n^{\circ} 1$ is obtained with 16 emission lines and iterations (spectral samples) 10 to 20 .

The best result for Weld test $n^{\circ} 2$ was achieved by considering 20 lines, and again with spectral samples 10 to 20 . These results suggest the employment of this sample window for calibration purposes, avoiding the initial samples which seem to be affected by the already discussed initial instability. It is also interesting to point out that the use of 10 or 20 plasma emission lines gives rise to a small difference in terms of the mean error estimated $(0.038712$ and 0.039866 , respectively) .

Table 3. Study of the best calibration polynomial obtained via automatic spectral calibration (experimental test).

\begin{tabular}{ccc}
\hline Weld test $\mathrm{n}^{\mathrm{o}}$ 1 (AISI-304) & \\
\hline Number of lines & $\begin{array}{c}\text { Iterations } \\
\text { (spectral samples) }\end{array}$ & Mean error (nm) \\
\hline 0 & 0 & 0.054234 \\
10 & $10-20$ & 0.045999 \\
20 & $0-10$ & 0.058072 \\
20 & $0-15$ & 0.037211 \\
20 & $10-30$ & 0.037554 \\
14 & $10-20$ & 0.041569 \\
16 & $10-20$ & 0.037117 \\
18 & $10-20$ & 0.038023 \\
Weld test $\mathrm{n}^{\mathrm{o}} 2$ (AISI-304) & & \\
\hline 0 & 0 & 0.049647 \\
20 & $10-20$ & 0.039866 \\
20 & $0-10$ & 0.048247 \\
15 & $10-20$ & 0.043649 \\
10 & $10-20$ & 0.038712 \\
\hline
\end{tabular}

An additional study was performed by means of the data acquired during a field test where an orbital process was considered for the tube-to-tubesheet arc-welding applied to steam generators [7]. The main interest in this case comes from the difference to be found between the spectra obtained in the experimental and field tests. An example of an experimental plasma spectrum was presented in figure 4, where a substantial continuum contribution can be observed. Figure 6 depicts four different spectra captured during the field tests. They do not exhibit a significant background radiation, and it should be also pointed out that the materials under analysis are different (AISI-304 in the experimental and INCONEL alloy in the field tests). The dissimilarities between the spectra can be mainly produced by the different geometry an input optics considered in each case. The four spectra of figure 6 have been included to show how they are clearly varying as the welding process evolves. Spectral samples 1, 5, 10 and 20 have been selected. It is worth noting that the main welding parameters, i.e. welding current and gas flow rate, are kept constant until the process ends, and the spectra dissimilarities are inherent to the arc-welding process.

The studies listed in Table 4 show how, again, the automatic calibration process allows to achieve a new polynomial with a better performance in terms of the estimated error. In this case sub-pixel algorithms have been included in the 
peak (emission line) modelling stage. In fact, the use of CDA (centroid detection algorithm) [3], provides the best result, particularly when 15 or 20 lines and spectral samples 10 to 20 are employed.
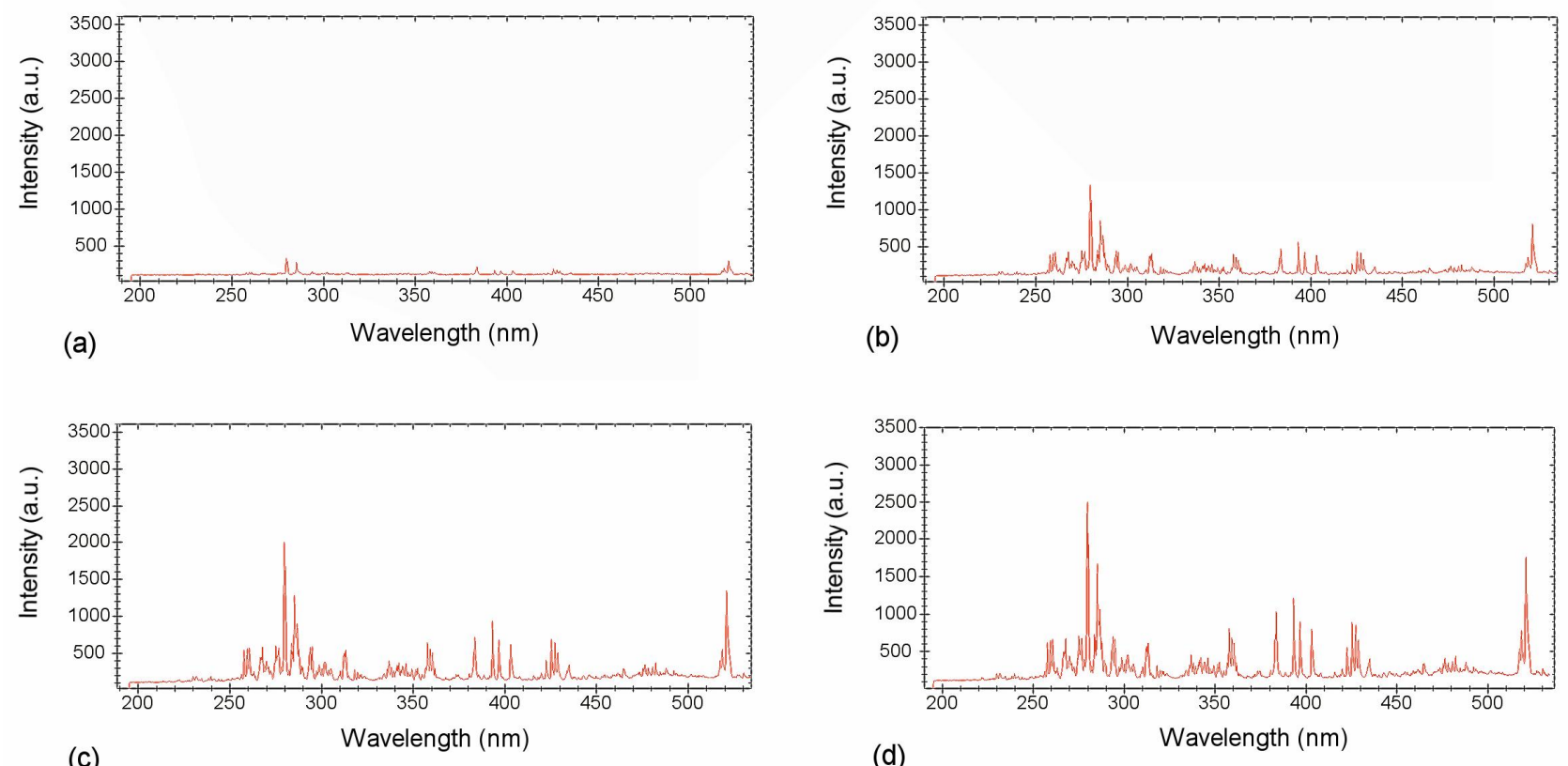

Fig. 6. Plasma spectra captured during field tests: (a) spectral capture $n^{\circ} 1$; (b) spectral capture $n^{\circ}$ 5; (c) spectral capture $n^{\circ}$ 10 ; (d) spectral capture $\mathrm{n}^{\mathrm{o}} 20$.

Table 4. Study of the best calibration polynomial obtained via automatic spectral calibration (field test).

Weld test $\mathrm{n}^{\mathrm{o}} 3$ (INCONEL alloy)

Number of lines Iterations (spectral samples) Sub-pixel Mean error (nm)

\begin{tabular}{cccc}
\hline 0 & 0 & No & 0.054216 \\
10 & $0-10$ & No & 0.051173 \\
10 & $0-20$ & No & 0.050718 \\
15 & $10-20$ & No & 0.048066 \\
20 & $10-20$ & LPO & 0.048684 \\
20 & $10-20$ & GF & 0.048325 \\
20 & $10-20$ & CDA & 0.043659 \\
10 & $10-20$ & CDA & 0.043757 \\
15 & $10-20$ & CDA & 0.043659 \\
\hline
\end{tabular}




\section{CONCLUSIONS}

In this paper we propose an on-line automatic wavelength calibration for CCD-spectrometers based on a processing scheme designed to be used as an on-line weld quality monitoring system. The typical solution to perform the required periodic wavelength calibrations of CCD-spectrometers lies in the use of calibration lamps, what not only implies an additional cost to the system, but also an off-line procedure. Given that the calculation of the plasma electronic temperature (to be used as a spectroscopic monitoring parameter) implies the identification of a specific set of plasma emission lines, and that the central wavelengths of these lines will be known in the process, they can be also used to developed and automatic in-process wavelength calibration, thus avoiding the employment of any additional light source. Initially, a new polynomial has been determined by performing a Gaussian-fit to the known lines emitted by a calibration lamp, improving the accuracy of the polynomial provided by the manufacturer. Experimental welding tests have been also conducted to check the performance of the proposed technique, demonstrating that, if a suitable number of plasma emission lines and spectral samples are chosen, a better polynomial in terms of the resulting mean error is obtained. In addition, the technique has also been checked with plasma spectra acquired during field tests, thus extending the validity of the proposed solution. In this case sub-pixel algorithms have also been included in the study, showing that their use in the emission line modelling stage can help finding the optimum polynomial. The relevance of using a best polynomial for the weld monitoring system can be significant, as it will imply that the resulting temperature profiles are more stable, thus enabling a better correlation with the possible appearance of defects along the seams.

\section{ACKNOWLEDGEMENTS}

This work has been co-supported by the Spanish TEC'2005-08218-C02-02 and TEC'2007-67987-C02-01 projects. Authors also want to thank J.J. Valdiande for his valuable collaboration in the development of the TIG welding tests.

\section{REFERENCES}

[1] Sforza, P. and de Blasiis, D., "On-line optical monitoring system for arc welding," NDT\&E Int. 35, 37-43 (2002).

[2] Ancona, A., Spagnolo, V., Lugara, P.M. and Ferrara, M., "Optical sensor for real-time monitoring of CO2 laser welding process," Appl. Opt. 40, 6019-6025 (2001).

[3] Mirapeix, J., Cobo, A., Conde, O.M., Jaúregui, C. and López-Higuera, J.M., "Fast algorithm for spectral processing with application to on-line welding quality assurance," Meas. Sci. Technol. 17(10), 2623-2629 (2006).

[4] National Institute for Standards and Technology (NIST) atomic spectra database (http://physics.nist.gov/cgibin/AtData/main_asd).

[5] Press, W.H., Teukolsky, S.A., Vetterling, W.T. and Flannery, B.P., "Numerical Recipes in C: The Art of Scientific Computing", Cambridge University Press, New York (1995).

[6] Systat software Inc., http://www.systat.com/products/PeakFit.

[7] Cobo, A., Mirapeix, J., Linares, F., Piney, J.A., Solana, D., and Lopez-Higuera, J.M., "Spectroscopic Sensor System for Quality Assurance of the Tube-To-Tubesheet Welding Process in Nuclear Steam Generators, " IEEE Sensors J. 7, 1219-24 (2007). 